\title{
Impact of chronic obstructive pulmonary disease on immune checkpoint inhibitor efficacy in advanced lung cancer and the potential prognostic factors
}

\author{
Jiebai Zhou ${ }^{1 \#}$, Yencheng Chao ${ }^{1 \#}$, Danwei Yao ${ }^{2}$, Ning Ding ${ }^{1}$, Jiamin Li $^{1}$, Lei Gao ${ }^{1}$, Yong Zhang", \\ Xiaobo Xu ${ }^{1}$, Jian Zhou $^{1}$, Balazs Halmos ${ }^{3}$, Nikolaos Tsoukalas ${ }^{4}$, Yuki Kataoka ${ }^{5}$, Ramon Andrade de Mello ${ }^{6}$, \\ Yuanlin Song ${ }^{1}$, Jie $\mathrm{Hu}^{1}$
}

${ }^{1}$ Department of Pulmonary Medicine, Zhongshan Hospital, Fudan University, Shanghai, China; ${ }^{2}$ Department of Epidemiology, Johns Hopkins University Bloomberg School of Public Health, Baltimore, USA; ${ }^{3}$ Department of Oncology, Montefiore Medical Center, Albert Einstein College of Medicine, Bronx, NY, USA; ${ }^{4}$ Department of Oncology, 401 General Military Hospital, Athens, Greece; ${ }^{5}$ Department of Respiratory Medicine, Hyogo Prefectural Amagasaki General Medical Center, Amagasaki, Japan; ${ }^{6}$ Faculdade de Medicina Universidade Nove de Julho (UNINOVE), Rua Pedro Oliveira Tavares, Bauru, SP, Brazil

Contributions: (I) Conception and design: J Hu, Y Song; (II) Administrative support: B Halmos, N Tsoukalas, Y Kataoka, RA de Mello; (III) Provision of study materials or patients: N Ding, J Li, Y Zhang, X Xu; (IV) Collection and assembly of data: J Zhou, Y Chao; (V) Data analysis and interpretation: J Zhou, D Yao; (VI) Manuscript writing: All authors; (VII) Final approval of manuscript: All authors.

\#These authors contributed equally to this work.

Correspondence to: Jie Hu. Department of Pulmonary Medicine, Zhongshan Hospital, Fudan University, 180 Fenglin Road, Shanghai 200032, China. Email: hu.jie@zs-hospital.sh.cn; Yuanlin Song. Department of Pulmonary Medicine, Zhongshan Hospital, Fudan University, 180 Fenglin Road, Shanghai 200032, China. Email: song.yuanlin@zs-hospital.sh.cn.

Background: The coexistence of chronic obstructive pulmonary disease (COPD) in lung cancer patients
often correlates with a poor clinical outcome regardless of tumor stage, mainly due to older age, poor lung
function, and complex comorbid disease. Emerging data suggest that the pathogenesis of both diseases
involves aberrant immune functioning. We conducted this retrospective study to describe the impact of
COPD on the clinical outcomes of lung cancer patients treated with immunotherapy and investigate the
potential prognostic factors. Methods: In total, 156 patients with advanced-stage lung cancer who received at least one administration of an anti-programmed cell death 1 (PD-1)/anti-programmed cell death-ligand 1 (PD-L1) immune checkpoint inhibitor (ICI) at any treatment line at Zhongshan Hospital Fudan University between May 2018 and December 2019 were enrolled in our study. Overall survival (OS) and progression-free survival (PFS) were analyzed according to the presence of COPD. We also evaluated the prognostic value of circulating cytokine levels for clinical outcome.

Results: We found that the presence of COPD (both spirometry-based COPD and physician-defined COPD) was significantly associated with longer PFS (316 vs. 186 days, $\mathrm{P}=0.018$ ). Moderate and severe COPD tended to have a better impact on the survival of these patients. In the present study, we reported that patients with mixed ventilatory defects tended to have a better OS $(\mathrm{P}=0.043)$ and $\mathrm{PFS}(\mathrm{P}=0.18)$ when treated with ICIs compared to the normal lung function group. We also found that low baseline plasma interleukin (IL)-8 and IL-2 receptor (IL-2R) levels were associated with longer PFS in patients with advanced-stage lung cancer who received ICI treatment. Furthermore, patients who had increased IL-2R levels had significantly poorer OS [hazard ratio $(\mathrm{HR})=3.63 ; 95 \%$ confidence interval $(\mathrm{CI}), 0.98-13.44 ; \mathrm{P}=0.040$ ] and $\mathrm{PFS}$ $(\mathrm{HR}=3.241$; 95\% CI, 1.032-10.18; P=0.035) when treated with ICIs. Nomograms were established based on the independent prognostic factors derived from our final multivariate models.

Conclusions: COPD was associated with better survival in advanced-stage lung cancer patients treated with ICIs. Plasma IL-8 and IL-2R levels were potential prognostic factors for clinical outcome. The 
nomograms represent a possibly useful tool for predicting the clinical outcomes of immunotherapy.

Keywords: Chronic obstructive pulmonary disease (COPD); immune checkpoint inhibitor (ICI); advanced lung cancer; mixed ventilatory defect; interleukin-8 (IL-8); interleukin-2R (IL-2R)

Submitted Sep 24, 2020. Accepted for publication Apr 27, 2021.

doi: $10.21037 /$ tlcr-21-214

View this article at: http://dx.doi.org/10.21037/tlcr-21-214

\section{Introduction}

Chronic obstructive pulmonary disease (COPD) is a common comorbidity in lung cancer, and is reported to affect $40-70 \%$ of lung cancer patients (1). In patients with moderate-to-severe COPD, the prevalence of lung cancer can reach as high as five times that of smokers without the disease (2). COPD is considered as an important independent risk factor for lung cancer, by increasing oxidative stress and the resulting DNA damage and repression of the DNA repair mechanisms, chronic exposure to pro-inflammatory cytokines, repression of innate immunity and increased cellular proliferation. T helper type 1 (Th1) cell population expands in both lung and tumor microenvironments in patients with non-small cell lung cancer (NSCLC). The generation of an immunosuppressive microenvironment allows tumor formation and progression, through which COPD might contribute to lung cancer development (3). The role of immunotherapy in the treatment of advanced-stage lung cancer is promising and rapidly evolving. Immune checkpoint inhibitors (ICIs) promote recognition of cancer cells as foreign cells by the immune system and reverse the tumor-driven inhibition of the immune system that promotes tumor growth. Clinical trials using antibodies to programmed cell death 1 (PD-1) and programmed cell death-ligand 1 (PD-L1) have demonstrated significant survival benefits in advanced NSCLC $(4,5)$, as well as in extensive-stage (ES)-small cell lung cancer (SCLC) (6). Previous studies report that respiratory symptoms, such as dyspnea, chronic cough, sputum production, and changes in pulmonary function, serving as diagnostic criteria for COPD, have a very negative impact on the prognosis of lung cancer patients (7-9). Mounting data suggests that the expression of immune checkpoint proteins PD-1 and PD-L1 is dysregulated in COPD patients (10-12). COPD-associated lung cancer might respond better to immunotherapy (13).

Considering the interesting but very limited literature in this area, we planned to conduct a retrospective study with the aim of describing the impact of COPD on the clinical outcomes of lung cancer patients treated with immunotherapy. Moreover, we explored promising prognostic biomarkers for the outcome of these patients treated with immunotherapy.

We present the following article in accordance with the STROBE reporting checklist (available at http://dx.doi. org/10.21037/tlcr-21-214).

\section{Methods}

\section{Study subjects and study approval}

The medical records of 188 consecutive patients with lung cancer who received at least one administration of an anti-PD-1/anti-PD-L1 ICI at any treatment line at Zhongshan Hospital Fudan University between May 2018 and December 2019 were retrospectively reviewed. Data were collected from our electronic medical record and patient follow-up. We excluded 13 patients for diagnosis of resectable lung cancer or concomitant synchronous metastatic malignancies aside from lung cancer, 19 patients with EGFR/ALK/ROS1 mutation. Finally, 156 evaluable patients were included.

This study was approved by the ethics committee of Zhongshan Hospital, Fudan University (No. B2017-142R). Informed consent from the patients included in this study was not required due to the retrospective nature of the study, and any personal information from the data was deleted beforehand. The study conformed to the provisions of the Declaration of Helsinki (as revised in 2013).

\section{Assessment of COPD}

Since only a subset of these patients $(62 / 156)$ had documented spirometry, we used available chest computed tomography (CT) imaging as well as spirometry to define the COPD and non-COPD subcohorts. For those patients with documented spirometry, we used the Global Initiative 
for Chronic Obstructive Lung Disease (GOLD) criteria to assess the presence of COPD and to evaluate the severity of airflow obstruction. COPD was defined as prebronchodilator forced expiratory volume in $1 \mathrm{~s} \mathrm{(FEV1)/}$ forced vital capacity $(\mathrm{FVC})<0.70$. The severity of airflow obstruction was staged by the GOLD criteria: mild (GOLD 1 , FEV1 $\geq 80 \%$ predicted), moderate (GOLD 2, 50\% $\leq$ FEV1 $<80 \%$ predicted), severe (GOLD 3, 30\% 5 FEV1 $<50 \%$ predicted), or very severe (GOLD 4, FEV1 $<30 \%$ predicted) (14).

According to the GOLD criteria, COPD was diagnosed in 31 patients and non-COPD in 31 patients. Three patients were classified as having mild COPD, 21 patients as having moderate COPD, and six patients as having severe COPD. For those patients without documented spirometry, we used clinical manifestation and chest CT imaging to diagnose COPD. For patients with complaints of chronic cough, sputum production and exertional dyspnea, their CT imagings were reviewed to detect widespread emphysema and exclude alternative diagnosis. Of the 94 patients without documented spirometry, 34 patients were diagnosed with COPD according to clinical manifestation and widespread emphysema on chest CT imaging.

\section{Assessment of ventilatory defect}

An obstructive ventilatory defect was defined as a FEV1/ FVC $<92 \%$ predicted. A restrictive ventilatory defect was defined as a total lung capacity (TLC) $<80 \%$ predicted and a FVC $<80 \%$ predicted. A mixed ventilatory defect was defined as presence of both obstructive and restrictive patterns. Of the 62 patients with documented spirometry, 19 patients had obstructive ventilatory defects, 12 patients had restrictive ventilatory defects, and 11 patients had mixed ventilatory defects.

\section{Data collection}

The data collected for all patients included age, sex, smoking history, pack-years index, performance status (PS), tumor-node-metastasis (TNM) staging, histology, PD-L1 expression, spirometric values, treatment regimens, survival, and adverse events.

\section{Evaluation of responses and toxicity}

We evaluated the overall survival (OS) and progression-free survival (PFS). OS was measured as the period from the initiation of immunotherapy to death. PFS was measured as the period from the first administration of immunotherapy to documented disease progression or death from any cause. Treatment response was assessed using the Response Evaluation Criteria in Solid Tumors (RECIST) version 1.1. The censored date was Aug 17, 2020.

\section{Statistical analysis}

We conducted completed case analysis. We focused on the patients with no missing data. The missing data in our sample was considered missing completely randomly. Some observations of patients were not recorded by chance, physicians sometimes forgot to enter into the computer so we didn't have a record of it.

Continuous data were presented as mean [standard deviation (SD) or median interquartile range (IQR)]. Chi-squared or Fisher's exact tests was used to compare categorical variables across subgroups. Statistical comparisons between continuous variables were performed with an independent Student's $t$-test for normally distributed data; otherwise, the Mann-Whitney $U$ test or Kruskal-Wallis Test was used. In order to better detect the association between baseline cytokine levels and antitumor activity, we used median values or time dependent receiver operating characteristic (ROC) curves analysis to specify the cut-offs for interleukin (IL)-8 and IL-2 receptor (IL-2R). The cumulative rate of OS or PFS was estimated using the Kaplan-Meier method with log-rank test. Time to events were measured from the date of the start of treatment until death or last visit.

Univariate and multivariate analyses were performed using Cox proportional hazards models to investigate the risk factors associated with fatal outcomes. The hazard ratio (HR) was reported along with the $95 \%$ confidence interval (CI). A two-sided $\mathrm{P}$ value $<0.05$ was considered statistically significant. Rms package (15) in R version 3.3.1 was applied to build the nomograms based on independent prognostic variables identified by multivariate Cox regression analyses. The performances of our nomograms were evaluated by Harrell concordance index (C-index). 1000 bootstrap resamples were used for internal validation of the accuracy of the predictions. Calibration curves were subsequently drawn to assess the consistency between the predicted probability and the actual proportion. All statistical analyses were performed using $\mathrm{R}$ software 
(version 3.3.1).

\section{Results}

\section{Patient characteristics}

Among the cohort of patients with advanced lung cancer, 31 cases were diagnosed with COPD according to the GOLD criteria, 34 cases had physician-diagnosed COPD, and 91 did not have COPD. Detailed demographic, therapeutic, and clinical information is presented in Table 1 . Age, sex, and smoking history were significantly different between the advanced lung cancer with COPD group and the advanced lung cancer without COPD cohort. Among the 116 patients in which PD-L1 expression was assessed, $\geq 50 \%$ of tumor cells exhibited PD-L1 expression in 30 patients $(25.9 \%)$, while no PD-L1 expression was observed in 27 patients (23.2\%). There were no significant differences in PD-L1 expression, PS at initiation of ICI therapy, therapeutic regimen, and treatment line between the COPD group and non-COPD group.

\section{Risk factors associated with death and progression}

Multivariable Cox proportional hazards regression analysis revealed that second-line immunotherapy (adjusted HR, 2.068 ; 95\% CI, 1.01-4.21) was significant risk factors associated with death in advanced lung cancer patients. PD-L1 expression of $\geq 50 \%$ (adjusted HR, $0.20 ; 95 \%$ CI, 0.061-0.64) was associated with lower death in advanced lung cancer patients (Table 2, Figure 1A). Second-line immunotherapy (adjusted HR, 2.31; 95\% CI, 1.36-3.93) was prone to have decreased progression free time during immunotherapy. PD-L1 expression of $\geq 50 \%$ (adjusted HR, 0.37; 95\% CI, 0.17-0.83) and diagnosed with COPD (adjusted HR, 0.56; 95\% CI, 0.33-0.96) were associated with a lower risk of disease progression (Table 3, Figure 1B).

\section{Treatment outcomes of ICI in patients with and without COPD}

The adjusted HR was statistically significant for the presence of COPD (adjusted HR, 0.56; 95\% CI, 0.33-0.96; $\mathrm{P}=0.034$ ) in the multivariate Cox proportional hazard model adjusting for the most relevant variables. The Kaplan-Meier curves for COPD (including spirometry-defined COPD and physician-diagnosed COPD) and non-COPD patients are presented in Figure 2. The median OS in non-COPD patients was 510 days, while the median OS in COPD patients was non-reached. COPD patients tended to have a longer OS (Figure 2A). The median PFS in COPD and non-COPD patients was 316 and 186 days, respectively, and the difference was statistically significant (adjusted HR, 0.56; 95\% CI, 0.33-0.96; $\mathrm{P}=0.018$ ) (Figure 2B).

\section{Treatment outcomes of ICI according to COPD severity}

For patients with spirometry-defined COPD, the KaplanMeier curves are shown in Figure 3. COPD patients had a longer median OS (532 vs. 495 days, $\mathrm{P}=0.93$, Figure $3 A$ ) and median PFS (278 vs. 189 days, $\mathrm{P}=0.20$, Figure $3 B$ ) compared to non-COPD patients, however the differences did not reach significance. Those with moderate and severe COPD tended to have better OS (553 vs. 495 days, $\mathrm{P}=0.54$, Figure $4 A$ ) and PFS (278 vs. 189 days, $\mathrm{P}=0.4$, Figure $4 B$ ), as compared to the non-COPD group.

\section{Treatment outcomes of ICI according to the different types of ventilatory defects}

When OS and PFS based on ventilatory defect types were compared to the normal lung function group, patients with mixed ventilatory defects tended to have better OS $(\mathrm{P}=0.043$, Figure 5A) and $\mathrm{PFS}(\mathrm{P}=0.18$, Figure 5B).

\section{Plasma cytokines associated with prognosis}

Part of our patients had dynamic serum cytokine data during ICI therapy. Based on the available data, we evaluated the impact of baseline or on-treatment changes in cytokines on the outcomes of ICI therapy in our cohort. We determined that $7 \mathrm{pg} / \mathrm{mL}$ is a clinically relevant threshold using ROC curve analysis. The IL-8 subsets were divided into low and high groups according to the corresponding cut-off level. Low baseline levels of plasma IL-8 in patients with advanced-stage lung cancer were associated with longer PFS (HR $=0.32 ; 95 \%$ CI, 0.07-1.36; $\mathrm{P}=0.087 ;$ Figure $6 A$ ). Area under the ROC Curve of IL-2R is less than 0.5 (0.417), so we instead selected median value $622 \mathrm{U} / \mathrm{mL}$ as the cutoff for IL-2R. Low baseline plasma IL-2R was significantly associated with longer PFS in advanced-stage lung cancer patients treated with ICIs (HR $=2.21 ; 95 \%$ CI, 1.01-4.81; $\mathrm{P}=0.042$; Figure $6 B$ ). These results suggested that low IL-8 and low IL-2R might serve as good prognostic biomarkers for the PFS of patients with advanced-stage lung cancer.

Next, we evaluated the dynamic changes of IL-2R 
Table 1 Demographic and clinical characteristics of patients with advanced-stage lung cancer

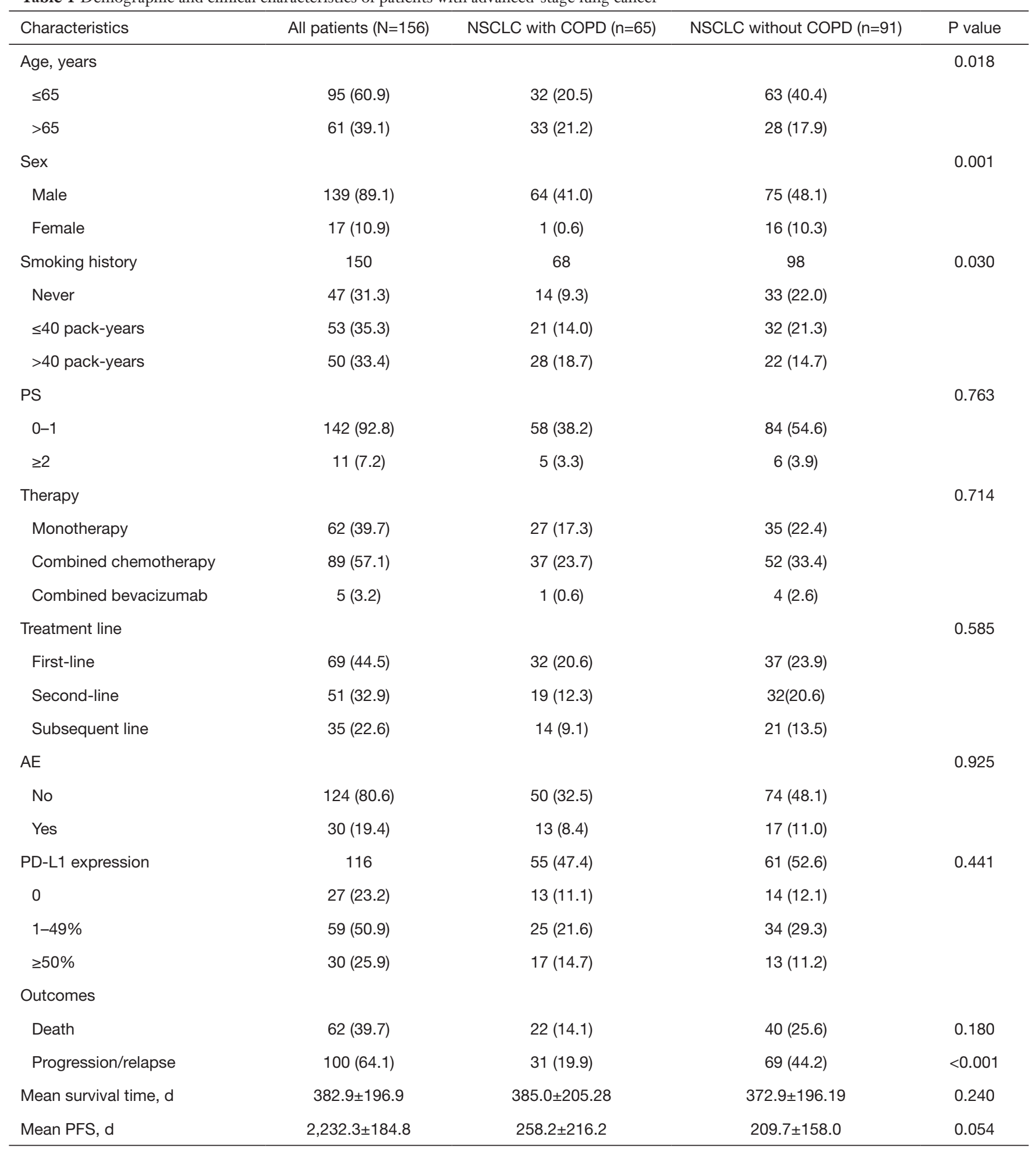

Data are presented as mean \pm SD or number (\%). P values comparing non-COPD and COPD cases are from the Chi-squared test, Fisher's exact test, or Mann-Whitney $U$ test. NSCLC, non-small cell lung cancer; COPD, chronic obstructive pulmonary disease; AE, adverse events; PS, performance status; PD-L1, programmed cell death-ligand 1; PFS, progression-free survival. 
Table 2 Cox regression analysis of the association between risk factors and death in patients with advanced-stage lung cancer

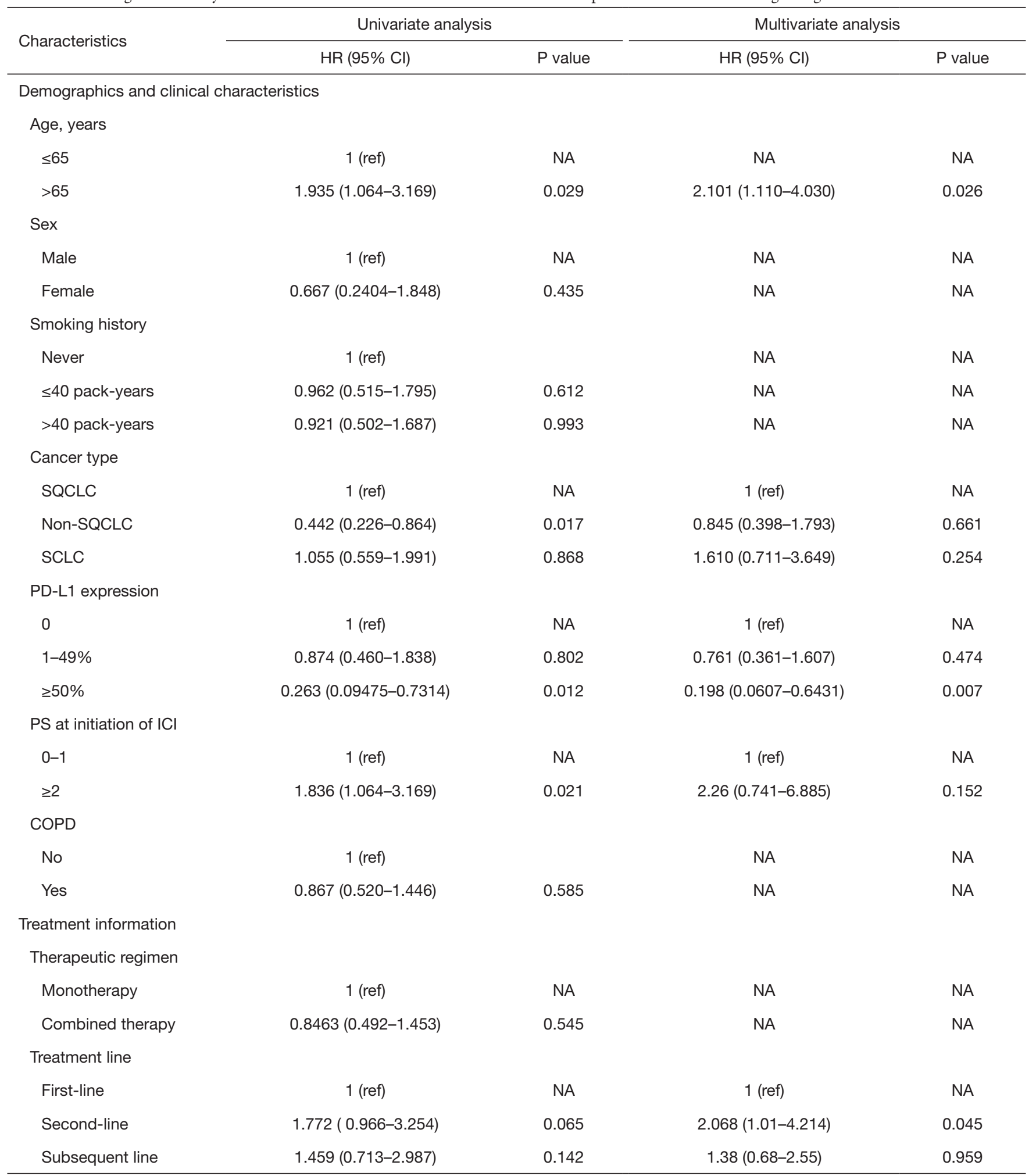

$\mathrm{HR}$, hazard ratio; Cl, confidence interval; SQCLC, squamous cell lung cancer; SCLC, small cell lung cancer; PD-L1, programmed cell death-ligand $1 ; \mathrm{ICl}$, immune checkpoint inhibitor. 


$\begin{array}{lcc}\text { A } & & \\ \text { Variable } & \text { Count } & \text { Precent (\%) } \\ \text { Age } & & \\ \leq 65 & 95 & 61 \\ >65 & 61 & 39 \\ \text { Cancer type } & & \\ \text { SQCLC } & 52 & 33 \\ \text { Non-SQCLC } & 72 & 46 \\ \text { SCLC } & 32 & 21 \\ \text { PDL1 Expression } & & \\ 0 \text { (ref) } & 27 & 23 \\ \text { 1-49\% } & 59 & 51 \\ \leq 50 \% & 30 & 26 \\ \text { Lines } & & \\ \text { First-line (ref) } & 69 & 44 \\ \text { Second-line } & 51 & 33 \\ \text { Subsequent line } & 35 & 23 \\ \text { PS } & & \\ 0-1 \text { (ref) } & 141 & 92 \\ >1 & 11 & 8\end{array}$

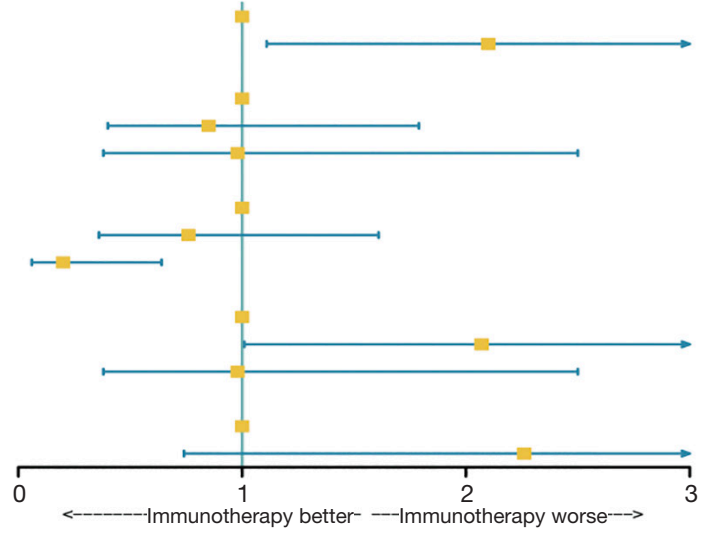

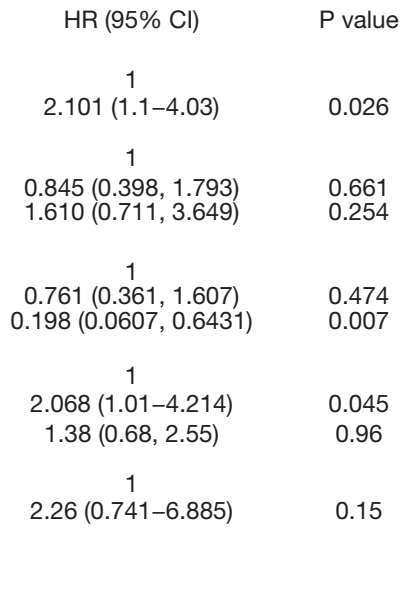

B

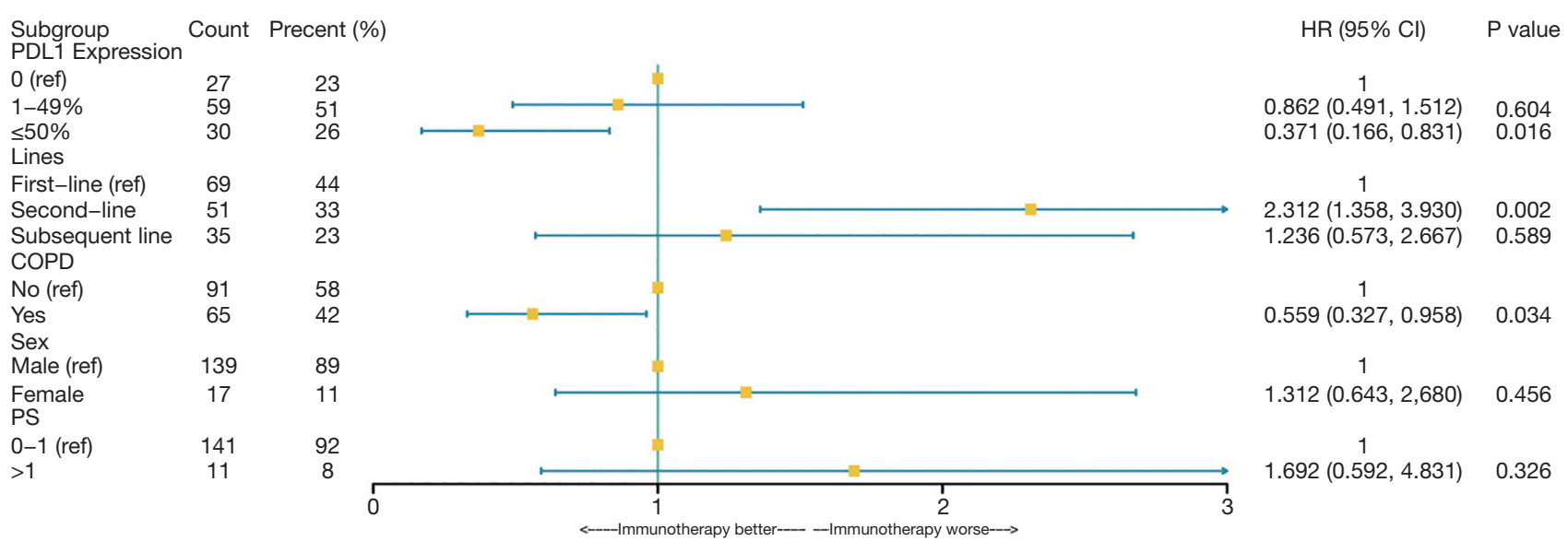

Figure 1 Risk factors of the clinical outcomes in the multivariate Cox proportional hazards regression model. (A) Forest plots present hazard ratios for OS of patients diagnosed with advanced-stage lung cancer. (B) Forest plots present hazard ratios for PFS of patients diagnosed with advanced-stage lung cancer. PS, performance status; CI, confidence interval; HR, hazard ratio; OS, overall survival; PFS, progressionfree survival.

levels following the first ICI administration. Changes in IL-2R levels were divided into two groups: (I) increase in value, and (II) no increase in value. Interestingly, patients who had increased IL-2R levels had significantly poorer OS (HR $=3.63 ; 95 \%$ CI, 0.98-13.44; $\mathrm{P}=0.040$; Figure 6C) and $\mathrm{PFS}(\mathrm{HR}=3.241 ; 95 \% \mathrm{CI}, 1.032-10.18 ; \mathrm{P}=0.035$; Figure 6D).

\section{Nomogram construction and validation}

Nomograms were established based on the independent prognostic factors derived from our final multivariate models (Figure 7). The OS nomogram included the following variables: PD-L1 expression, treatment line, PS, age, and cancer type (Figure $7 A$ ). For the predicted PFS nomogram, we further added sex and COPD variables without compromising the discriminative ability of the model (Figure $7 B$ ). The nomograms shed light on the important contribution of PD-L1 expression and treatment line for advanced-stage lung cancer, while PS and sex conferred a moderate influence on survival. One hundred was set as the maximum score of each variable; by adding up the total score and locating it on the bottom point scale, an estimated probability of survival could be easily determined. 
Table 3 Cox regression analysis of the association between risk factors and progression in patients with advanced-stage lung cancer

\begin{tabular}{|c|c|c|c|c|}
\hline Characteristics & \multicolumn{2}{|c|}{ Univariate analysis } & \multicolumn{2}{|c|}{ Multivariate analysis } \\
\hline \multicolumn{5}{|c|}{ Demographics and clinical characteristics } \\
\hline \multicolumn{5}{|l|}{ Age, years } \\
\hline$\leq 65$ & 1 (ref) & NA & NA & NA \\
\hline \multicolumn{5}{|l|}{ Sex } \\
\hline Male & 1 (ref) & NA & 1 (ref) & NA \\
\hline Female & $1.816(1.025-3.218)$ & 0.059 & $1.312(0.643,2.680)$ & 0.456 \\
\hline \multicolumn{5}{|l|}{ Smoking history } \\
\hline$>40$ pack-years & $1.033(0.6224-1.715)$ & 0.900 & NA & NA \\
\hline \multicolumn{5}{|l|}{ Cancer type } \\
\hline SQCLC & 1 (ref) & NA & NA & NA \\
\hline Non-SQCLC & $1.026(0.635-1.656)$ & 0.918 & NA & NA \\
\hline SCLC & $1.535(0.905-2.602)$ & 0.112 & NA & NA \\
\hline \multicolumn{5}{|l|}{ PD-L1 expression } \\
\hline 0 & 1 (ref) & NA & 1 (ref) & NA \\
\hline $1-49 \%$ & $0.965(0.558-1.670)$ & 0.899 & $0.862(0.491-1.512)$ & 0.604 \\
\hline No & 1 (ref) & NA & 1 (ref) & NA \\
\hline Yes & $0.597(0.388-0.920)$ & 0.019 & $0.559(0.327-0.958)$ & 0.034 \\
\hline \multicolumn{5}{|l|}{ Treatment information } \\
\hline \multicolumn{5}{|l|}{ Therapeutic regimen } \\
\hline Monotherapy & 1 (ref) & NA & NA & NA \\
\hline Combined therapy & $0.7301(0.499-1.070)$ & 0.107 & NA & NA \\
\hline \multicolumn{5}{|l|}{ Treatment line } \\
\hline First-line & 1 (ref) & NA & 1 (ref) & NA \\
\hline Second-line & $1.990(1.250-3.160)$ & 0.004 & $2.312(1.358-3.930)$ & 0.002 \\
\hline Subsequent line & $1.771(1.040-3.010)$ & 0.034 & $1.236(0.573-2.667)$ & 0.589 \\
\hline
\end{tabular}

$\mathrm{HR}$, hazard ratio; Cl, confidence interval; SQCLC, squamous cell lung cancer; SCLC, small cell lung cancer; PD-L1, programmed cell death-ligand 1; ICl, immune checkpoint inhibitor; COPD, chronic obstructive pulmonary disease. 
A

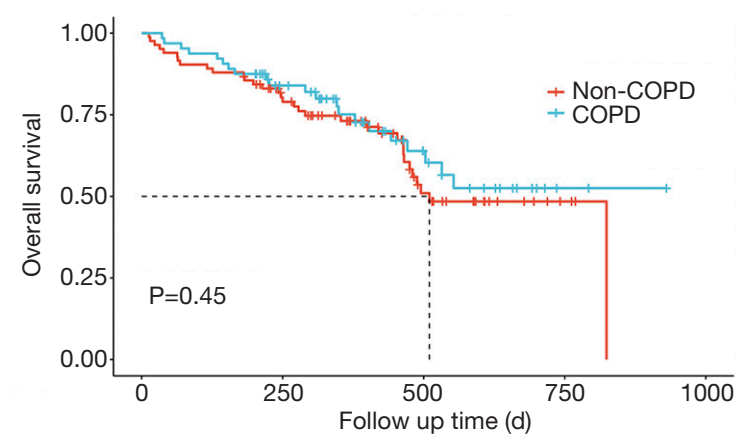

Number at risk

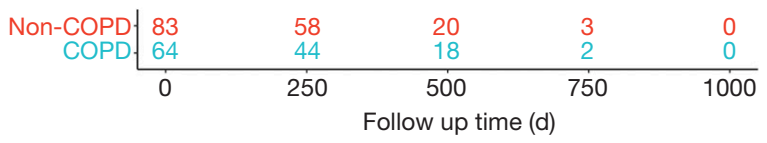

B

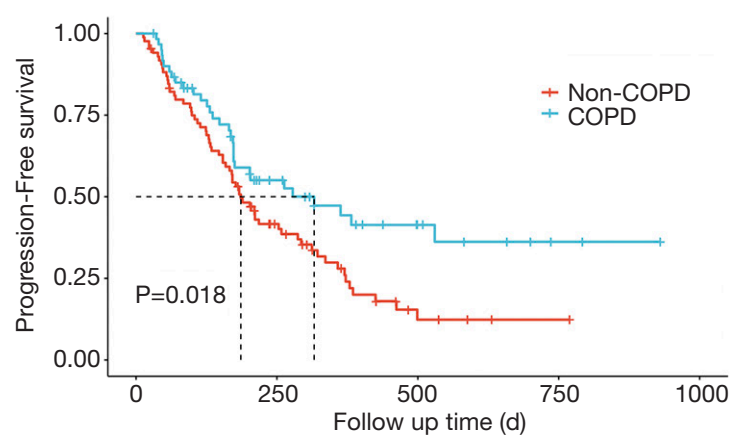

Number at risk

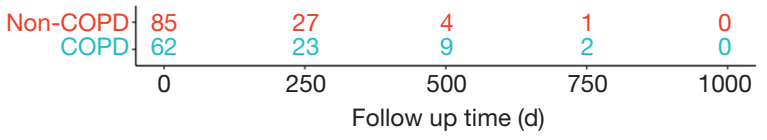

Figure 2 Treatment outcomes of ICIs in patients with and without COPD. (A) Kaplan-Meier curve of OS by COPD (including spirometrydefined COPD and physician-diagnosed COPD). (B) Kaplan-Meier analysis of the PFS probability by COPD. The dotted lines indicate median survival time. Patients with COPD appear to gain longer survival time, and were significantly associated with better PFS in our cohort (316 vs. 186 days; adjusted HR, 0.56; 95\% CI, 0.33-0.96; P=0.018). COPD, chronic obstructive pulmonary disease; ICI, immune checkpoint inhibitor; OS, overall survival; PFS, progression-free survival; HR, hazard ratio; CI, confidence interval.

A

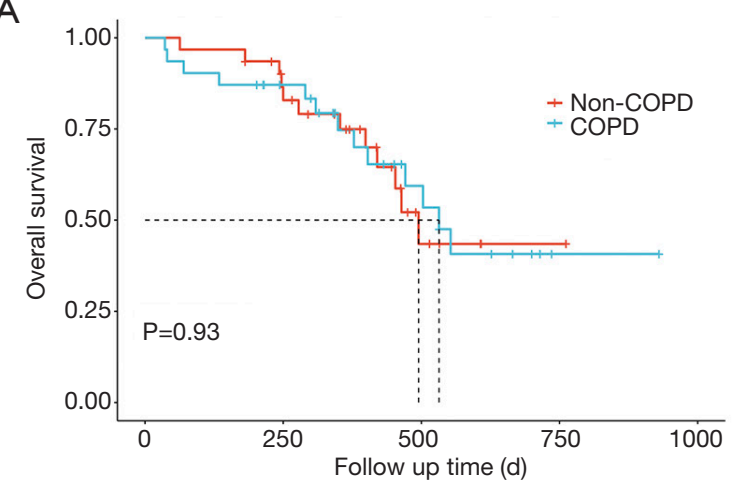

Number at risk

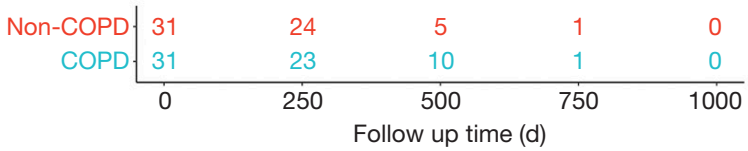

B

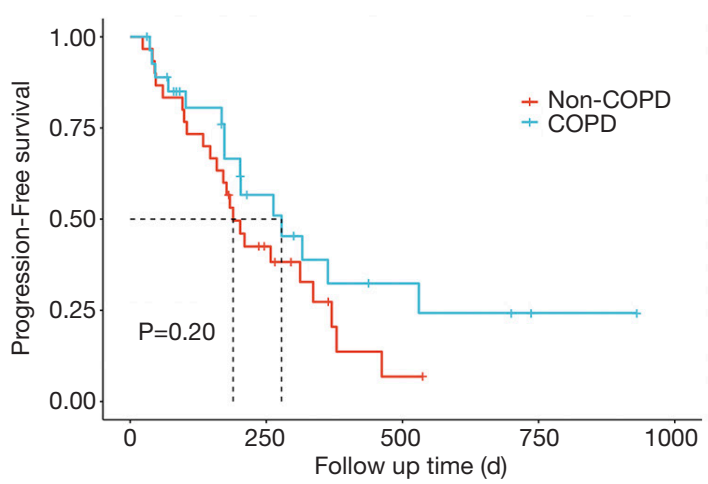

Number at risk

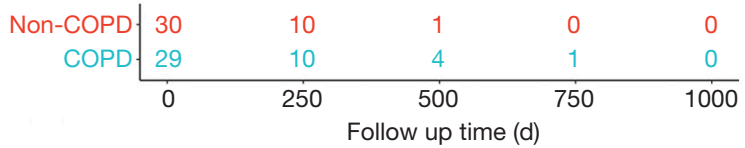

Figure 3 Treatment outcomes of ICIs in patients with and without spirometry-defined COPD. (A) Kaplan-Meier curve of OS by spirometry-defined COPD. (B) Kaplan-Meier analysis of the PFS probability by spirometry-defined COPD. COPD patients had a longer median OS (532 vs. 495 days, $\mathrm{P}=0.93)$ and median PFS (278 vs. 189 days, $\mathrm{P}=0.20)$ compared to non-COPD patients, however the differences did not reach significance. COPD, chronic obstructive pulmonary disease; ICI, immune checkpoint inhibitor; OS, overall survival; PFS, progression-free survival.

Calibration plots of both nomograms are displayed in the Figures S1,S2. The C-index of the built nomogram to predict OS $(0.687 ; 95 \%$ CI, 0.679-0.695), and that of the second nomogram $(0.681 ; 95 \%$ CI, 0.674-0.697) both demonstrate an optimal consistency between the nomogram prediction and the actual observation for advanced-stage 
A

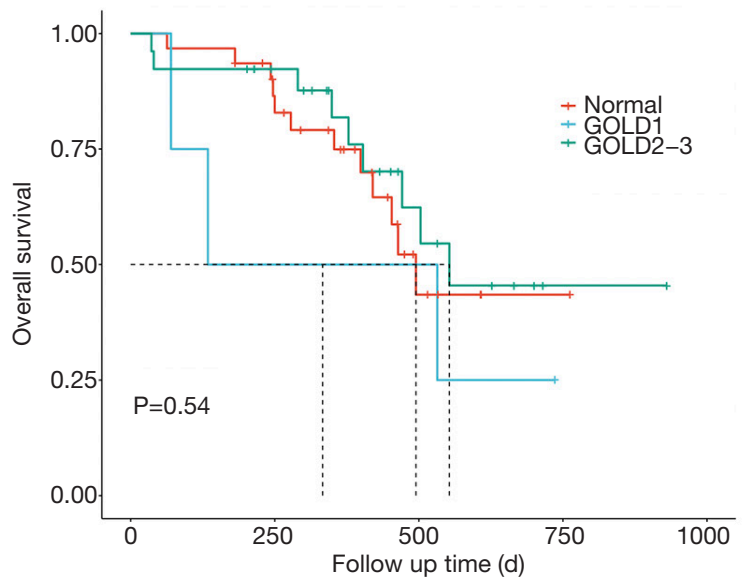

B

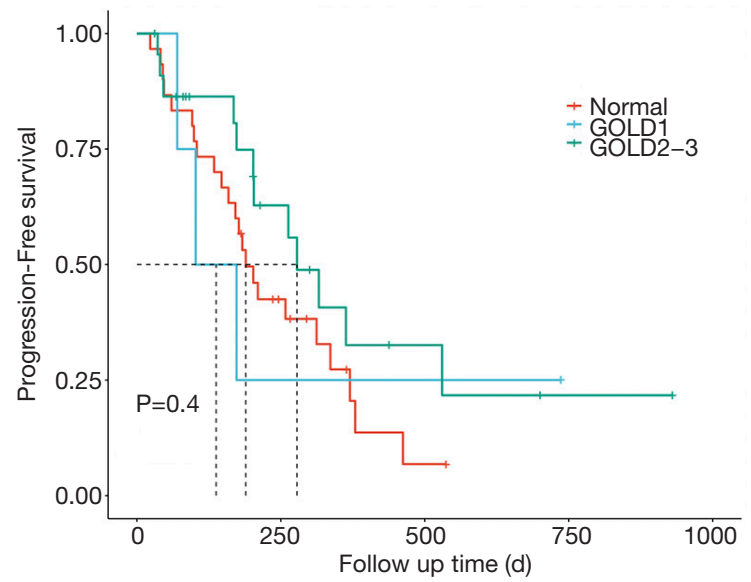

Figure 4 Treatment outcome of ICIs according to COPD severity. (A) Kaplan-Meier curve of OS by COPD severity. (B) Kaplan-Meier analysis of the PFS probability by COPD severity. Risk group stratification within COPD severity. Those with moderate and severe COPD tended to have better OS (553 vs. 495 days, $\mathrm{P}=0.54$ ) and PFS ( $278 v$ vs. 189 days, $\mathrm{P}=0.4$ ) as compared to the non-COPD group. GOLD 1, mild COPD; GOLD 2, moderate COPD; GOLD 3, severe COPD; ICI, immune checkpoint inhibitor; COPD, chronic obstructive pulmonary disease; OS, overall survival; PFS, progression-free survival.
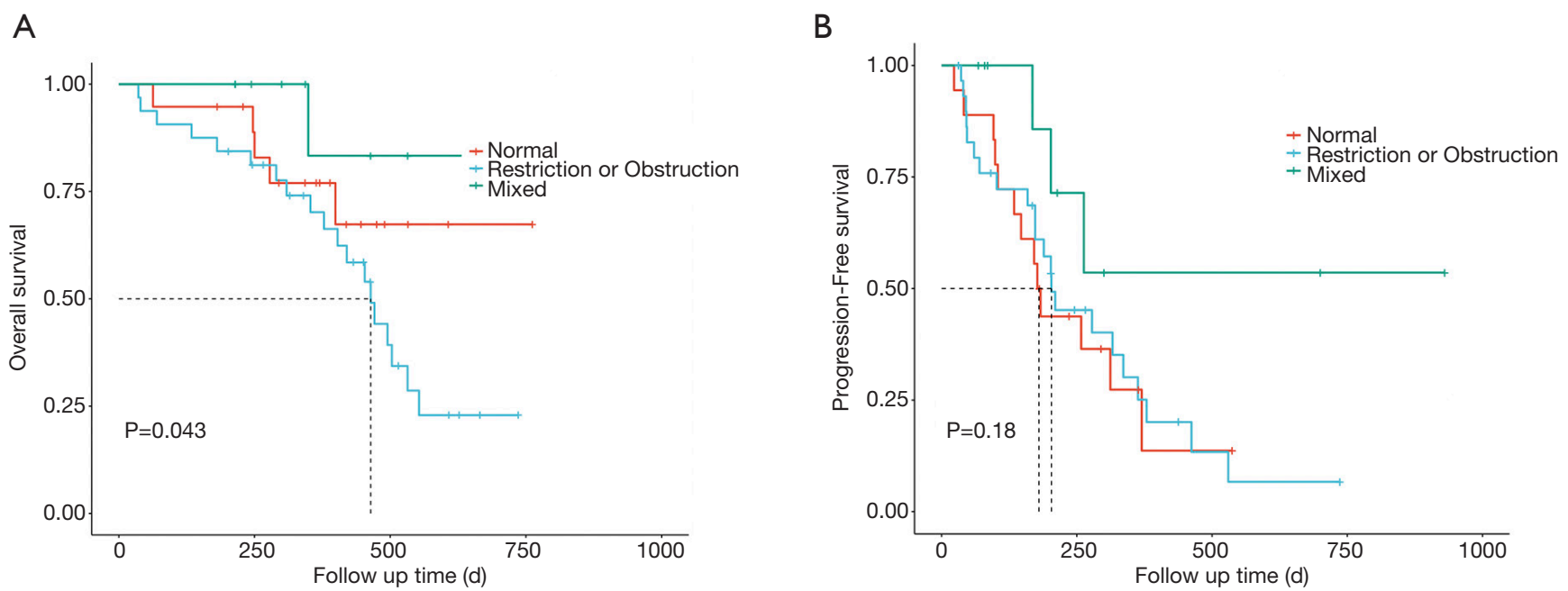

Figure 5 Treatment outcome of ICIs according to different types of ventilatory defects. (A) Kaplan-Meier analysis of OS in the cohort stratification of risk groups based on the types of ventilatory defects. (B) Kaplan-Meier analysis of PFS in the cohort stratification of risk groups based on the types of ventilatory defects. ICI, immune checkpoint inhibitor; OS, overall survival; PFS, progression-free survival.

lung cancer patients.

\section{Discussion}

COPD is a common comorbidity in lung cancer. Young et al. reported that among newly diagnosed lung cancer cases, the prevalence of COPD was six times higher than that of matched smokers (16). Patients with COPD have more symptoms than people without COPD, such as cough, sputum production, and dyspnea. Also, quality of life in patients with COPD was markedly decreased in several aspects. The coexistence of COPD itself often prevents patients from receiving the optimal lung cancer treatment due to older age, poor lung function, and complex 
A

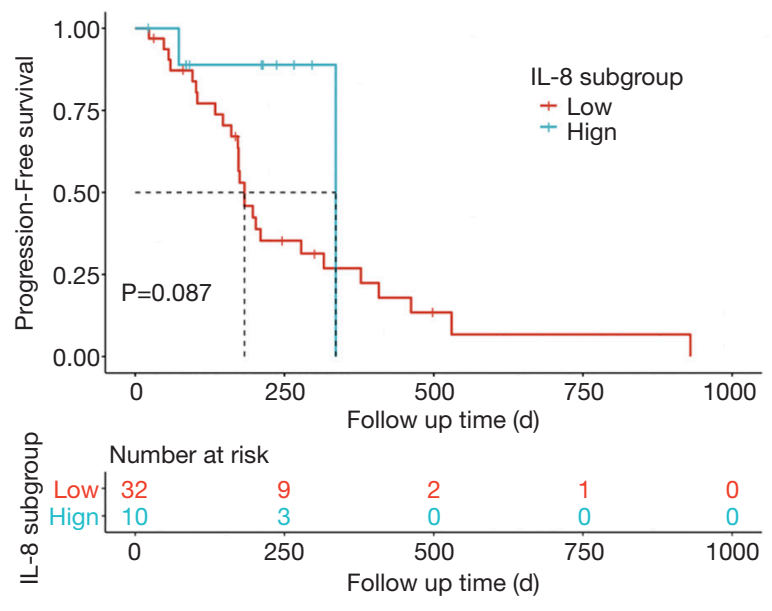

C

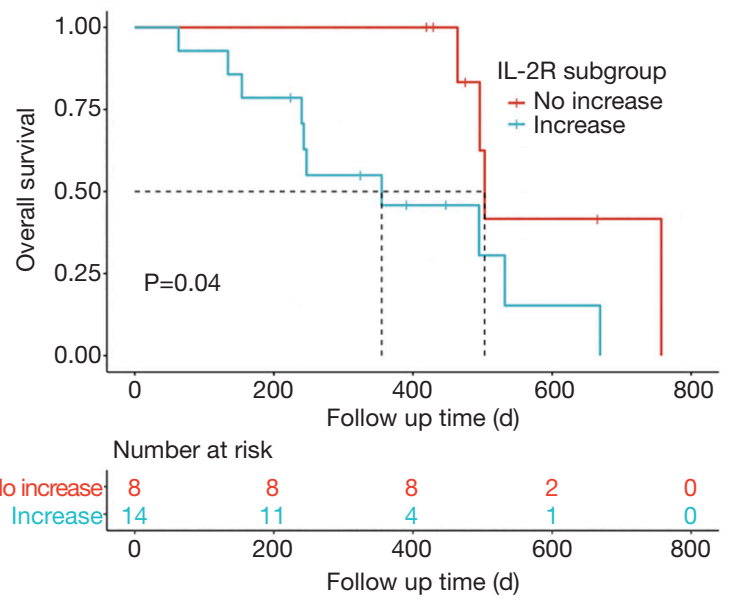

B
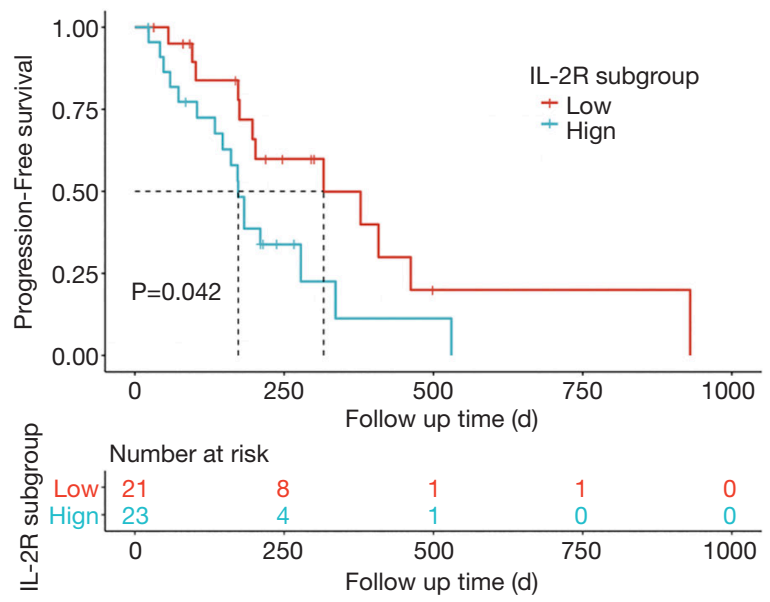

D

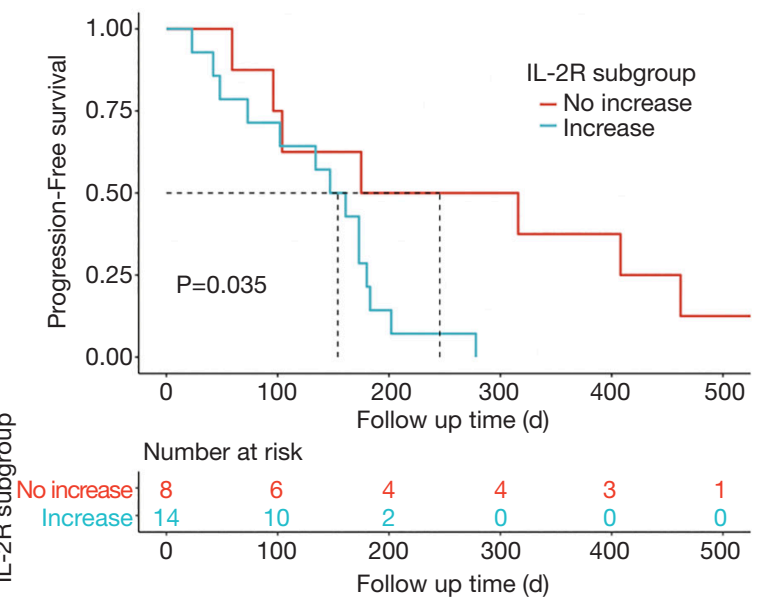

Figure 6 Plasma cytokines associated with prognosis. (A) Association between baseline IL-8 level and PFS. Patients were stratified using an IL-8 level of $7 \mathrm{pg} / \mathrm{mL}$. Low baseline plasma IL-8 levels (median cut-off $7 \mathrm{pg} / \mathrm{mL}$ ) tended to have longer PFS in cohort, though these were not statistically significant (HR $=0.32 ; 95 \% \mathrm{CI}, 0.07-1.36$ ); $\mathrm{P}=0.087$ ); (B) low baseline IL-2R level (cut-off, $622 \mathrm{U} / \mathrm{mL}$ ) was associated with longer PFS ( $\mathrm{HR}=2.21 ; 95 \% \mathrm{CI}, 1.01-4.81 ; \mathrm{P}=0.042$ ). Two-sided log-rank test $\mathrm{P}$ value, the number of patients with available IL-8 and IL-2R are shown; (C,D) on-treatment changes in plasma IL-2R and outcomes in patients with available cytokines levels; (C) on-treatment increases in plasma IL-2R were significantly associated with worse OS in our cohort (HR =3.63; 95\% CI, 0.98-13.44; $\mathrm{P}=0.040$ ); (D) on-treatment increases in plasma IL-2R were also associated with worse PFS according to our data (HR =3.241; 95\% CI, 1.032-10.18; P=0.035). IL, interleukin; OS, overall survival; PFS, progression-free survival; HR, hazard ratio; CI, confidence interval.

comorbidities. The concomitance of COPD in lung cancer patients really portends a poor clinical outcome, regardless of tumor stage (17-20). In addition, patients with NSCLC and COPD have a low incidence of oncogenic driver mutations (21), indicating that targeted therapies are less likely to improve survival.

In the present study of patients with advanced-stage lung cancer who received ICI therapy, the presence of COPD was significantly associated with longer PFS, which is in accordance with previous studies $(9,22,23)$. The subgroup analysis revealed that moderate and severe COPD tended to have a better impact on the survival of these patients, though these differences did not reach significance.

The chronic inflammation associated with COPD may contribute to the earliest stages of carcinogenesis (24). The immune system plays an important role in the 
A

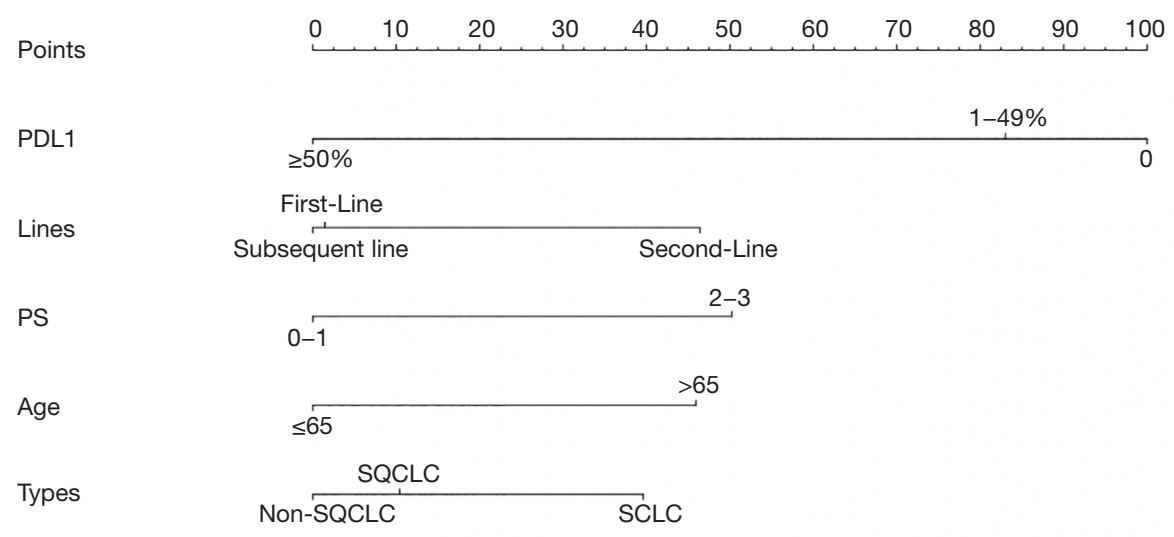

Total points

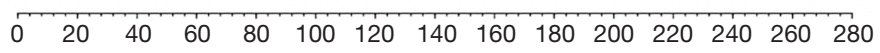

Probablity of 1-year OS

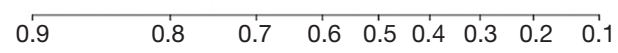

B

Points

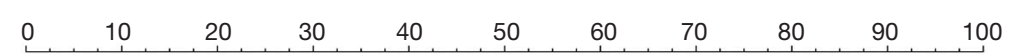

Sex

PDL1

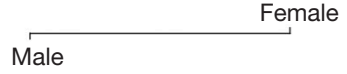

COPD

$\geq 50 \%$

$1-49 \%$

Lines

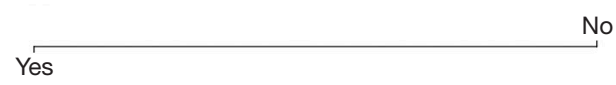

Subsequent line

PS

First-Line

Second-Line

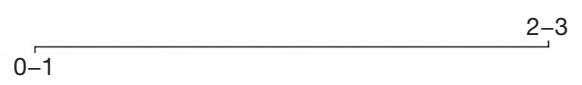

Total points

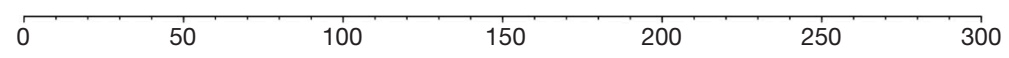

Probablity of 1-year OS

$\begin{array}{llllllll}0.7 & 0.6 & 0.5 & 0.4 & 0.3 & 0.2 & 0.1 & 0.05\end{array}$

Figure 7 Nomograms. (A,B) Prognostic nomograms for predicting the 1-year OS and PFS probabilities of patients with advanced-stage lung cancer. Prognostic value is located on each variable axis, and a line is drawn upward to determine the number of point nomograms for predicting the probabilities of patients. OS, overall survival; PD-L1, programmed cell death-ligand 1; COPD, chronic obstructive pulmonary disease; PS, performance status; PFS, progression-free survival.

establishment of chronic inflammation in COPD and the control of tumor burden in lung cancer. However, the effect of COPD on the immune microenvironment of lung cancer remains unclear, and its impact on the efficacy of ICIs remains unknown. Mark et al. (22) observed that patients with COPD had an increased fraction of cluster of differentiation (CD) 3, CD4+, and CD8+ cells in non- tumor lung tissue compared to smokers without COPD. They also importantly identified that Th1 cell populations were expanded in both lung and tumor microenvironments in patients with COPD. Furthermore, they found that there was increased expression of the immune checkpoints $\mathrm{T}$ cell immunoglobin and mucin-domain containing-3 (TIM3) and PD- 1 by $\mathrm{CD}^{+} \mathrm{T}$ cells in tumors from patients with 
COPD. Biton et al. (9) demonstrated higher levels of CD8+ tumor-infiltrating lymphocytes (TILs) exhaustion in fresh tumor samples. Exhausted T cells exhibit a dysfunctional tumor-specific immune response, with reduced cytokine production and decreased survival with increased expression of inhibitory receptors $(25,26)$. ICI therapy may reverse exhaustion and promote tumor regression in patients with cancer. Given the role of the immune system in both COPD and lung cancer, as well as the increased use of ICIs, a better understanding of the link between COPD and lung cancer is the fundation for improved treatment.

In the present study, we reported that patients with mixed ventilatory defects tended to have a better OS and PFS when treated with ICIs compared to the normal lung function group. Causes of restrictive lung disease may be intrinsic or extrinsic to the lung parenchyma. Examples of intrinsic causes are interstitial lung diseases, pneumonia, and surfactant deficiency, while empyema, chest deformities, respiratory muscle weakness, hemothorax, pneumothorax, and pleural effusion or thickening are examples of extrinsic causes. Obstructive pulmonary diseases include COPD, emphysema, bronchial asthma, chronic bronchitis, and bronchiectasis (27). For patients with mixed ventilatory defects, the combination of both defect patterns might physiologically contribute to a better clinical outcome of immunotherapy. However, the underlying molecular mechanisms still need to be investigated.

In addition to the factors we found to be associated with clinical outcomes of immunotherapy, including PS score, treatment line of immunotherapy, and PD-L1 expression, we also noticed that plasma cytokines could predict the efficacy of ICIs. Cytokines refer to a variety of peptides and glycoproteins secreted by immunocompetent cells and tumor cells, including growth factors such as IL, interferon, chemokines, and tumor necrosis factor. We found that low baseline plasma IL-8 levels were associated with longer PFS in patients with advanced-stage lung cancer who received ICI treatment, which is consistent with previous studies (28). IL-8 is a proinflammatory chemokine, which induces neutrophil degranulation (29). IL-8 may also promote tumor cell growth and metastasis through multiple mechanisms, including epithelial-to-mesenchymal transition (30), angiogenesis (31), formation of neutrophil extracellular traps (32), and infiltration of immunosuppressive and protumorigenic myeloid inflammatory cells that inhibit the function of antitumor cytotoxic T cells (31). The mechanistic basis of IL-8-associated resistance to immunotherapy requires further investigation.
Furthermore, we reported that patients with low baseline plasma IL-2R exhibited improved clinical outcomes when treated with ICIs. Interestingly, patients who had increased IL-2R levels had significantly poorer OS and PFS. IL-2 regulates $\mathrm{CD}^{+} \mathrm{T}$ cell differentiation and function, as well as $\mathrm{CD}^{+} \mathrm{T}$ cells effector and memory responses. The loss of or responsiveness to IL-2 at least partly explains the exhausted phenotype that occurs in tumor responses (33). As far as we know, there are no studies considering the effect of IL-2/ IL-2R on immunotherapy. The underling mechanism of the prognostic role of IL-2R in immunotherapy and whether IL-2/IL-2R could be a potential therapeutic target is still unclear.

We also established nomograms to predict clinical outcomes of patients receiving ICI therapy based on the independent prognostic factors derived from our final multivariate models. The nomograms shed light on the important contribution of PD-L1 expression and treatment line for advanced-stage lung cancer, while PS and sex conferred a moderate influence on survival. Validation of the nomogram is an essential step to avoid overfitting of the model. In this study, we conducted internal validation, and the calibration plots demonstrated optimal agreement between prediction and actual observation. However, external validation should also be conducted in order to guarantee the repeatability and reliability of the established nomograms.

Our study had several limitations to be discussed. Firstly, this was a retrospective cohort study conducted in a single center, which might limit the generalizability of the findings. Secondly, in the present study, physiciandiagnosed COPD might be related to a certain degree of misclassification. In addition, because of the limited number of patients with post-bronchodilator spirometry, we used pre-bronchodilator spirometry to define COPD, which might overestimate the COPD diagnosis. However, our data may be more valuable in a real-world clinical setting, since patients who receive immunotherapy for lung cancer do not undergo spirometry or post-bronchodilator spirometry routinely. Thirdly, due to the retrospective nature of our study, the lack of complete data about circulating cytokines for all patients may have led us to miss some prognostic cytokines. This also tempered our ability to draw strong conclusions regarding the role of cytokines in immunotherapy of advanced-stage lung cancer.

\section{Conclusions}

The present study confirmed that COPD was associated 
with better clinical outcomes in advanced-stage lung cancer patients treated with ICIs. Patients with mixed ventilatory defects tended to have better OS and PFS when treated with ICIs. The baseline and on-treatment changes in IL-8 and IL-2R were significantly associated with survival of patients who received immunotherapy, and may serve as convenient prognostic biomarkers. While more studies are needed, the work presented here promises to accelerate the development of potential precision medicine biomarkers for selecting advanced-stage lung cancer patients who are most likely to benefit from immunotherapy. The nomograms represent a possibly useful tool for predicting the clinical outcomes of immunotherapy.

\section{Acknowledgments}

The authors appreciate the academic support from AME Lung Cancer Collaborative Group. And also thank the study participants and their families, and are grateful for the collaboration of Zhongshan Hospital, Fudan University, and their staff.

Funding: This research was supported by the Shanghai Municipal Key Clinical Specialty (shslczdzk02201), Natural Science Foundation of China (81770039), and Shanghai Top-priority Clinical Key Disciplines Construction Project (2017ZZ02013).

\section{Footnote}

Reporting Checklist: The authors have completed the STROBE reporting checklist. Available at http://dx.doi. org/10.21037/tlcr-21-214

Data Sharing Statement: Available at http://dx.doi. org/10.21037/tlcr-21-214

Conflicts of Interest: All authors have completed the ICMJE uniform disclosure form (available at http://dx.doi. org/10.21037/tlcr-21-214). The authors have no conflicts of interest to declare.

Ethical Statement: The authors are accountable for all aspects of the work in ensuring that questions related to the accuracy or integrity of any part of the work are appropriately investigated and resolved. This study was approved by the ethics committee of Zhongshan Hospital, Fudan University (No. B2017-142R). The requirement for informed consent from the patients included this study was waived due to the retrospective nature of the study, and any personal information from the data was removed beforehand. The study conformed to the provisions of the Declaration of Helsinki (as revised in 2013).

Open Access Statement: This is an Open Access article distributed in accordance with the Creative Commons Attribution-NonCommercial-NoDerivs 4.0 International License (CC BY-NC-ND 4.0), which permits the noncommercial replication and distribution of the article with the strict proviso that no changes or edits are made and the original work is properly cited (including links to both the formal publication through the relevant DOI and the license). See: https://creativecommons.org/licenses/by-nc-nd/4.0/.

\section{References}

1. Alberg AJ, Brock MV, Samet JM. Epidemiology of lung cancer: looking to the future. J Clin Oncol 2005;23:3175-85.

2. de Torres JP, Marín JM, Casanova C, et al. Lung cancer in patients with chronic obstructive pulmonary disease-incidence and predicting factors. Am J Respir Crit Care Med 2011;184:913-9.

3. Caramori G, Ruggeri P, Mumby S, et al. Molecular links between COPD and lung cancer: new targets for drug discovery? Expert Opin Ther Targets 2019;23:539-53.

4. Gandhi L, Rodríguez-Abreu D, Gadgeel S, et al. Pembrolizumab plus Chemotherapy in Metastatic NonSmall-Cell Lung Cancer. N Engl J Med 2018;378:2078-92.

5. Carbone DP, Reck M, Paz-Ares L, et al. First-Line Nivolumab in Stage IV or Recurrent Non-Small-Cell Lung Cancer. N Engl J Med 2017;376:2415-26.

6. Horn L, Mansfield AS, Szczęsna A, et al. First-Line Atezolizumab plus Chemotherapy in Extensive-Stage Small-Cell Lung Cancer. N Engl J Med 2018;379:2220-9.

7. Kalathil SG, Lugade AA, Pradhan V, et al. T-regulatory cells and programmed death $1+\mathrm{T}$ cells contribute to effector T-cell dysfunction in patients with chronic obstructive pulmonary disease. Am J Respir Crit Care Med 2014;190:40-50.

8. Bhat TA, Panzica L, Kalathil SG, et al. Immune Dysfunction in Patients with Chronic Obstructive Pulmonary Disease. Ann Am Thorac Soc 2015;12 Suppl 2:S169-75.

9. Biton J, Ouakrim H, Dechartres A, et al. Impaired Tumor-Infiltrating T Cells in Patients with Chronic Obstructive Pulmonary Disease Impact Lung Cancer 
Response to PD-1 Blockade. Am J Respir Crit Care Med 2018;198:928-40.

10. Yun YH, Kim YA, Sim JA, et al. Prognostic value of quality of life score in disease-free survivors of surgically-treated lung cancer. BMC Cancer 2016;16:505.

11. Grutters JP, Joore MA, Wiegman EM, et al. Healthrelated quality of life in patients surviving non-small cell lung cancer. Thorax 2010;65:903-7.

12. Ban WH, Lee JM, Ha JH, et al. Dyspnea as a Prognostic Factor in Patients with Non-Small Cell Lung Cancer. Yonsei Med J 2016;57:1063-9.

13. Narayanapillai SC, Han YH, Song JM, et al. Modulation of the PD-1/PD-L1 immune checkpoint axis during inflammation-associated lung tumorigenesis. Carcinogenesis 2020;41:1518-28.

14. GOLD. Global strategy for the diagnosis MaPoC, Global Initiative for Chronic Obstructive Lung Disease (GOLD) 2018. Available online: http://www.goldcopd.org/. Accessed April 4, 2018.

15. Frank E Harrell Jr (2017). rms: Regression Modeling Strategies. R package version 5.1-0. Available online: https://CRAN.R-project.org/package=rms

16. Young RP, Hopkins RJ, Christmas T, et al. COPD prevalence is increased in lung cancer, independent of age, sex and smoking history. Eur Respir J 2009;34:380-6.

17. Zhai R, Yu X, Shafer A, et al. The impact of coexisting COPD on survival of patients with early-stage non-small cell lung cancer undergoing surgical resection. Chest 2014;145:346-53.

18. Shin S, Park HY, Kim H, et al. Joint effect of airflow limitation and emphysema on postoperative outcomes in early-stage nonsmall cell lung cancer. Eur Respir J 2016;48:1743-50.

19. Gao YH, Guan WJ, Liu Q, et al. Impact of COPD and emphysema on survival of patients with lung cancer: A meta-analysis of observational studies. Respirology 2016;21:269-79.

20. Lim JU, Yeo CD, Rhee CK, et al. Overall survival of driver mutation-negative non-small cell lung cancer patients with COPD under chemotherapy compared to non-COPD non-small cell lung cancer patients. Int J Chron Obstruct Pulmon Dis 2018;13:2139-46.

21. Lim JU, Yeo CD, Rhee CK, et al. Chronic Obstructive Pulmonary Disease-Related Non-Small-Cell Lung Cancer Exhibits a Low Prevalence of EGFR and ALK Driver Mutations. PLoS One 2015;10:e0142306.

22. Mark NM, Kargl J, Busch SE, et al. Chronic Obstructive Pulmonary Disease Alters Immune Cell Composition and Immune Checkpoint Inhibitor Efficacy in NonSmall Cell Lung Cancer. Am J Respir Crit Care Med 2018;197:325-36.

23. Shin SH, Park HY, Im Y, et al. Improved treatment outcome of pembrolizumab in patients with nonsmall cell lung cancer and chronic obstructive pulmonary disease. Int J Cancer 2019;145:2433-9.

24. Zhu HX, Shi L, Zhang Y, et al. Myocyte enhancer factor 2D provides a cross-talk between chronic inflammation and lung cancer. J Transl Med 2017;15:65.

25. Wherry EJ, Kurachi M. Molecular and cellular insights into T cell exhaustion. Nat Rev Immunol 2015;15:486-99.

26. Thommen DS, Schreiner J, Müller P, et al. Progression of Lung Cancer Is Associated with Increased Dysfunction of T Cells Defined by Coexpression of Multiple Inhibitory Receptors. Cancer Immunol Res 2015;3:1344-55.

27. Lutfi MF. The physiological basis and clinical significance of lung volume measurements. Multidiscip Respir Med 2017;12:3.

28. Yuen KC, Liu LF, Gupta V, et al. High systemic and tumor-associated IL-8 correlates with reduced clinical benefit of PD-L1 blockade. Nat Med 2020;26:693-8.

29. Baggiolini M, Walz A, Kunkel SL. Neutrophil-activating peptide-1/interleukin 8 , a novel cytokine that activates neutrophils. J Clin Invest 1989;84:1045-9.

30. Fernando RI, Castillo MD, Litzinger M, et al. IL-8 signaling plays a critical role in the epithelial-mesenchymal transition of human carcinoma cells. Cancer Res 2011;71:5296-306.

31. David JM, Dominguez C, Hamilton DH, et al. The IL-8/IL-8R Axis: A Double Agent in Tumor Immune Resistance. Vaccines (Basel) 2016;4:22.

32. Alfaro C, Teijeira A, Onate C, et al. Tumor-Produced Interleukin-8 Attracts Human Myeloid-Derived Suppressor Cells and Elicits Extrusion of Neutrophil Extracellular Traps (NETs). Clin Cancer Res 2016;22:3924-36.

33. Spolski R, Li P, Leonard WJ. Biology and regulation of IL-2: from molecular mechanisms to human therapy. Nat Rev Immunol 2018;18:648-59.

Cite this article as: Zhou J, Chao Y, Yao D, Ding N, Li J, Gao L, Zhang Y, Xu X, Zhou J, Halmos B, Tsoukalas N, Kataoka Y, de Mello RA, Song Y, Hu J. Impact of chronic obstructive pulmonary disease on immune checkpoint inhibitor efficacy in advanced lung cancer and the potential prognostic factors. Transl Lung Cancer Res 2021;10(5):2148-2162. doi: 10.21037/ tlcr-21-214 


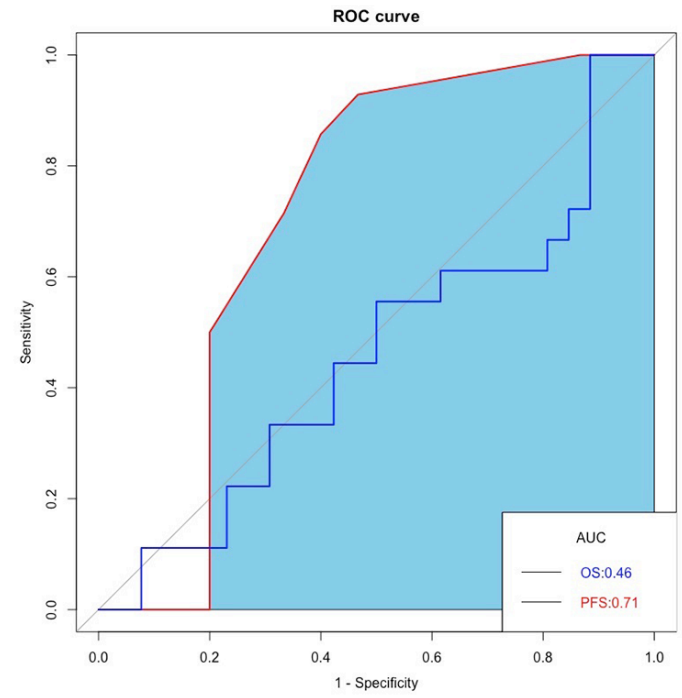

Figure S1 Statistical identification of a baseline IL-8 cutoff. ROC curves analyses on OS and PFS for all patients with available IL-8 levels. As the OS ROC curve obtained the highest area under the curve (AUC =0.71), the Youden's index (sensitivity + specifity-1) for the OS ROC curve was considered to select the threshold level as $7 \mathrm{pg} / \mathrm{mL}$.

A

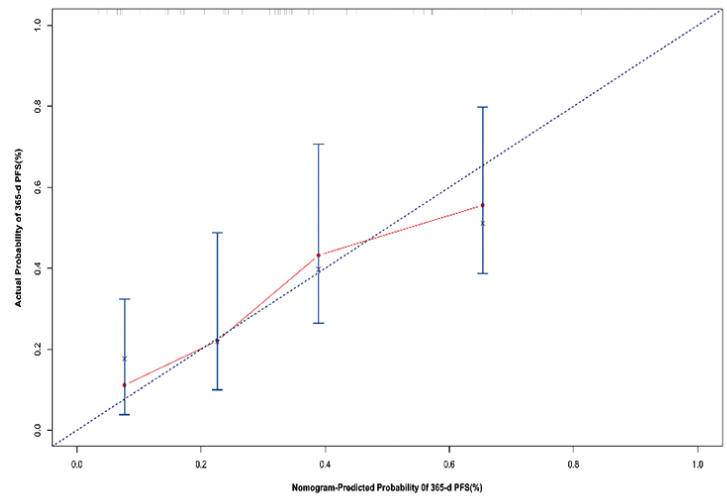

B

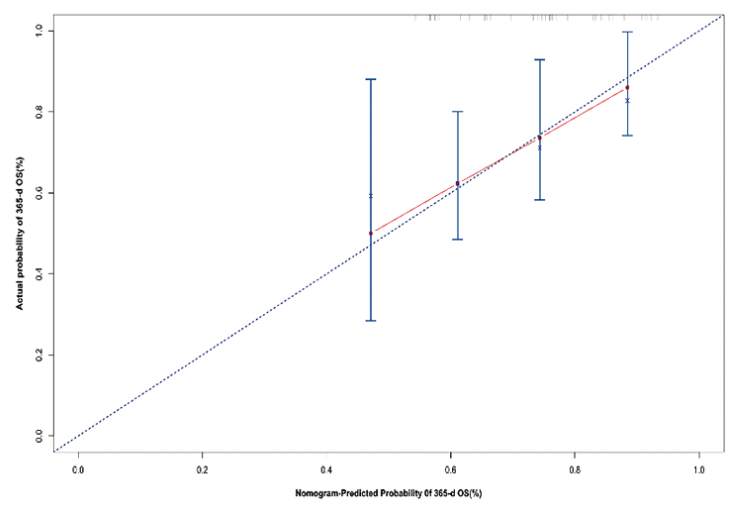

Figure S2 Calibration curves for predicting 1-year survival of patients. (A) One year overall survival; (B) one year progression-free survival. Nomogram-predicted survival is plotted on the $\mathrm{x}$-axis; actual survival probability is plotted on the $\mathrm{y}$-axis. A plot among 45 -degree line would indicate a perfect calibration model in which the predicted outcomes are identical to the actual outcomes. 